\title{
PSICOLOGÍA POSITIVA EN LAS AULAS DE MAGISTERIO CON BITÁCORA, DEBATE, ABP Y TEATRO
}

\author{
Elena Briones \\ Área de Psicología Evolutiva y de la Educación \\ Universidad de Cantabria. Facultad de Educación \\ elena.briones@unican.es \\ Alicia Gómez-Linares \\ Área de Psicología Evolutiva y de la Educación \\ Universidad de Cantabria. Facultad de Educación \\ alicia.gomez@unican.es \\ Raquel Palomera \\ Área de Psicología Evolutiva y de la Educación \\ Universidad de Cantabria. Facultad de Educación \\ raquel.palomera@unican.es
}

Fecha de Recepción: 8 Febrero 2019

Fecha de Admisión: 30 Abril 2019

\section{RESUMEN}

La educación en valores y competencias socio-emocionales ha sido reconocida como parte esencial de la formación inicial y permanente del profesorado. En este trabajo presentamos un programa diseñado desde el marco de la Psicología Positiva para la asignatura de Formación en Valores y Competencias Personales para Docentes (FVCPD) impartida en los dos Grados en Magisterio de la Universidad de Cantabria. Este programa, integra exposiciones teóricas con metodologías activas centradas en el aprendizaje experiencial, reflexivo y documentado en un cuaderno de bitácora, el aprendizaje dialógico, el basado en problemas (ABP) y el Teatro Foro. El alumnado participante respondió, anónima y voluntariamente, a la evaluación de su empatía, antes y después de la formación, y a una valoración final del proceso de enseñanza-aprendizaje. Los resultados reflejan la amplia satisfacción del alumnado con las innovadoras metodologías, sus aprendizajes (fundamentalmente en lo que respecta a la conciencia social, la autogestión emocional y la conciencia y valor de uno mismo), y permiten la identificación de elementos para la optimización del programa. Por una parte, la valoración realizada de los componentes esenciales de cada metodología sugiere cuál requiere de un mayor seguimiento compartido entre profesorado y alumnado. Por otra parte, el impacto evaluado sobre la empatía demuestra el potencial de las estrategias empleadas. Se concluye con la necesidad de seguir investigando con objeto de optimizar las herramientas metodológicas empleadas, para la docencia y para la evaluación. 
Palabras clave: competencias socio-emocionales; docentes en formación; educación emocional; formación en valores; psicología positiva

\section{ABSTRACT}

Positive psychology in the masters 'classrooms with bitácora, debate, abp and theatre. The education in values and socio-emotional competences has been recognized as an essential part of the initial part and permanent teacher training. In this work, we present a program designed from the framework of Positive Psychology for the subject named Training in Values and Personal Competences for Pre-Service Teachers (TVPCPT), taught in the two degrees in Teaching at the University of Cantabria. This program, integrates theoretical expositions with active methodologies centered on reflective and documented experiential learning in a logbook, dialogic learning, problem based learning (PBL) and Forum Theatre. The participating students responded, anonymously and voluntarily, to the evaluation of their empathy, before and after the training and to a final evaluation of the teaching-learning process. The results reflect the wide satisfaction of the students with the innovative methodologies, their learning (fundamentally with regard to social conscience, emotional self-management and self-awareness and its value) and allow the identification of elements for the optimization of the program.On the one hand, the assesment made of the essential components of each methodology suggests which requieres a greater shared monitoring between teachers and students.On the othe hand, the impact assesed on empathy demonstrates the potential of the strategies employed. It concludes with the need to continue researching in order to optimize the methodological tools used for teaching and for evaluation.

Keywords: socio-emotional competences; pre-service teachers; emotional education; teaching in values; positive psychology

\section{INTRODUCCIÓN}

El paradigma desde el cual nos aproximamos a la materia de Formación en Valores y Competencias Personales para Docentes (FVCPD) es el de la Psicología Positiva, puesto que se centra en el desarrollo, la promoción y la prevención de la salud y el bienestar (Seligman, Ernst, Gillham, Reivich y Linkins, 2009).

\section{Psicología positiva en la educación superior y en la formación docente}

Las escuelas saludables o positivas se caracterizan por su eficacia y eficiencia como organización, por la motivación y satisfacción de sus maestros y por el desarrollo académico, personal y social de sus alumnos (Moreno y Gálvez, 2010). De esta manera, la implicación, satisfacción, motivación y desarrollo profesional y humano de los docentes, suponen un pivote básico de las escuelas para contribuir a la salud y bienestar de todos sus integrantes. Desde este planteamiento se sostiene, además, que la creación de un ambiente de seguridad, confianza, vinculación e identidad escolar estimula al alumno a progresar, refuerza su autoestima, hace que se sienten mejor tratados y supone la base para la convivencia y posterior ciudadanía (Fernández Domínguez et al., 2009; Moreno y Gálvez, 2010; Pañellas, Alguacil, y Boqué, 2016; Seligman et al., 2009).

En consecuencia, la educación en valores y competencias socio-emocionales ha sido reconocida como parte esencial de la formación inicial y permanente del profesorado. El Libro Blanco del Título de Grado en Magisterio (ANECA, 2004) ha integrado estas competencias en la definición del perfil profesional del docente en el EEES, y aconseja su enseñanza mediante nuevas dinámicas instructivas. Además, es objetivo de la formación superior ofrecer una educación centrada en lo académico, lo profesional y lo vital, integrando así dos ejes principales: la formación teórica (para conocer y proyectar) y la formación personal vivenciada, destacando esta última, por favorecer la acción, 
la convivencia, el sentir y el ser. Por tanto, en la formación de maestros se persigue tanto su desarrollo personal como su preparación para educar emocionalmente a sus alumnos.

Esta educación socio-emocional conviene encuadrarla en un marco ético, que indique sus fines y facilite la construcción de los valores fundamentales en el contexto escolar. En palabras de Fernández Domínguez et al. (2009) este marco ético supone el eje vertebrador de la formación socio-emocional puesto que "en la educación emocional no se puede perder de vista que de la biología -las emociones- hay que pasar a la construcción ética. Es decir, partir de lo que somos para llegar a lo que deseamos ser". Lo cual a su vez requiere de un análisis profundo de elementos identitarios que conduzcan al autoconocimiento, la clarificación de valores y desarrollo de competencias tales como la flexibilidad cognitiva y el pensamiento crítico. Por tanto, se trata de garantizar una preparación de los maestros, que les ayude a ser personas más conscientes de sí mismas, más maduras y equilibradas, para que de esta manera influyan positivamente en sus educandos, y desde su propia solidez personal y formación les ayuden a fortalecerse y a ser ellos mismos, desplegando sus potencialidades (Fernández Domínguez, 2005).

\section{b) Programa de aprendizaje social y emocional para docentes en formación}

El objetivo principal de la asignatura FVCPD es proporcionar aprendizaje socio-emocional (ASE) siguiendo los criterios SAFE para el diseño de su formación (Secuenciación progresiva, metodologías Activas, Focalización en competencias concretas, Entrenamiento explícito) (Durlak et al., 2011), así como los principios propuestos para la formación con adultos (Kornacki y Caruso, 2007) centrados en favorecer: la valoración y reflexión, una experiencia guiada, la transferencia, el apoyo permanente y la evaluación continua.

Esta formación pretende promover el desarrollo de cinco competencias socio-emocionales (CES) identificadas como fundamentales para el maestro (e.g. CASEL, 2005; Fernández Domínguez et al., 2009): conciencia de uno mismo, autogestión, conciencia social, habilidades de relación, y toma de decisiones responsables.

La asignatura FVCPD se imparte en el módulo de formación básica de los dos Grados de Magisterio (i.e. Educación Infantil y Educación Primaria) en la Universidad de Cantabria (UC). Se cursa de manera presencial en el primer cuatrimestre (15 semanas) del primer curso, en dos sesiones semanales de dos horas cada una (6 ECTS), en clases constituidas por grupos desde 25 a personas. Las primeras dos semanas, el alumnado se organiza en pequeños grupos, con el objeto de realizar la tarea grupal. Al tiempo, tienen lugar diversas dinámicas, primero de cohesión grupal durante la presentación del marco teórico general por parte del profesorado; y a continuación, se van introduciendo de forma teórica las CES (véase el orden seguido en la Figura 1) acompañadas de procedimientos específicos que potencien la auto-valoración en cada competencia y su entrenamiento.

Además, el programa que aquí presentamos (véase cronograma en la Figura 1), integra diversas metodologías activas, articuladas en dos niveles: un primer nivel, destinado a la formación del yo-personal, centrado en el conocimiento y desarrollo de las propias competencias del alumnado; y en un segundo nivel, donde se transciende al yo-profesional, con situaciones futuras como docentes.

Las tareas que comprenden estos dos niveles, y que son objeto de evaluación consisten en un Cuaderno de bitácora y la aplicación del Teatro Foro. Mediante el Cuaderno de bitácora se plantean tres actividades:

Tarea 1 de meta-conocimiento, que consiste en realizar un análisis sobre la identificación de lo aprendido en cada tema y su aplicación estableciendo un plan de acción personal. 
Tarea 2 sobre clarificación de valores (propia y en situaciones de conflicto), argumentación dialógica y posicionamiento propio en un dilema moral habitual en estudiantes de Magisterio, construido al efecto por las docentes (véase una versión en Briones y Lara, 2016).

Tarea 3 para el estudio de caso mediante la metodología de Aprendizaje Basado en Problemas (ABP). Se analizan las CES en un caso propuesto por las docentes sobre una situación vivida por una estudiante de Magisterio; además deben proponer soluciones para optimizar la situación aplicando los conocimientos previamente adquiridos (véase una explicación en detalle en Briones y Gómez-Linares, 2016).

Figura 1. Cronograma de la asignatura FVCPD.

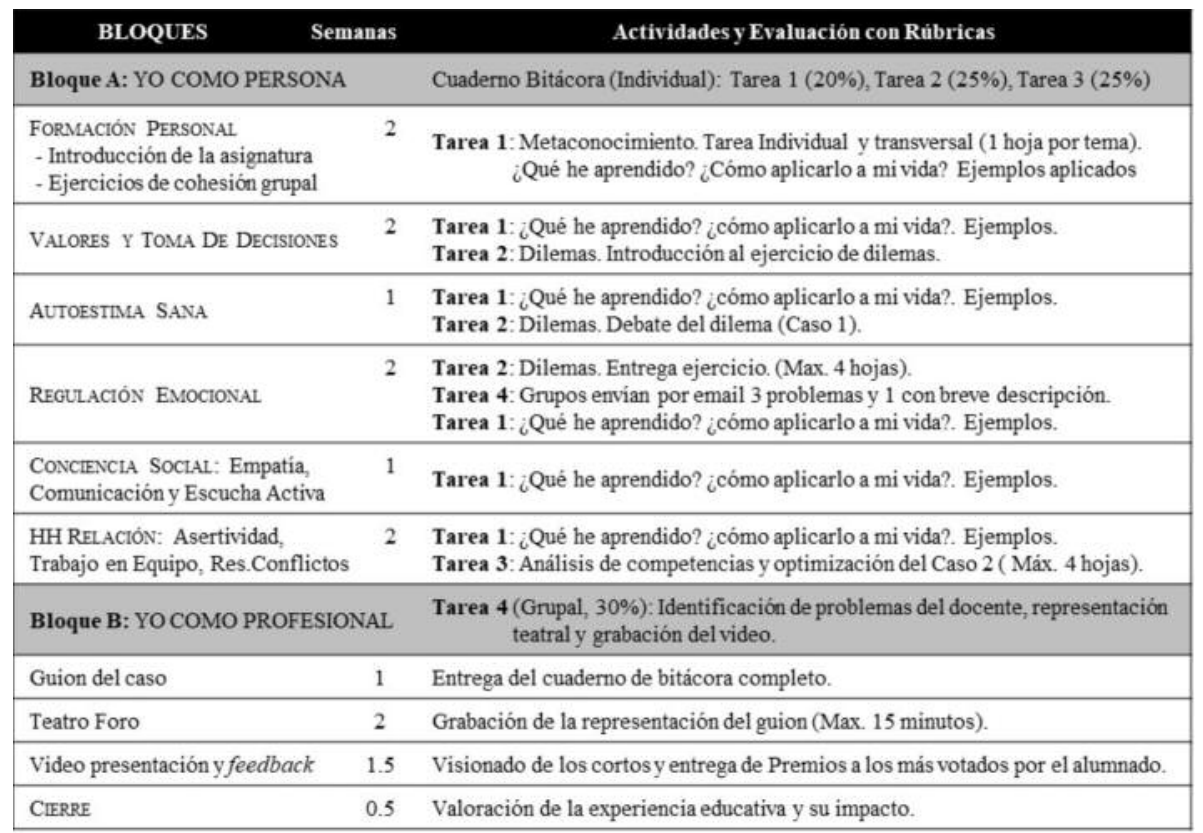

Por su parte mediante el Teatro Foro se pretende favorecer la asimilación de las CES. Para ello empleamos estrategias del Teatro del Oprimido de Augusto Boal (Motos-Teruel y Navarro-Amorós, 2012), buscando la reflexión sobre los problemas propios de los docentes, relacionados con el manejo de CES y dilemas morales en su día a día, al tiempo que se invita al alumnado a comprometerse en la búsqueda de una mejor solución y a su práctica vivencial (véase el proceso en la Figura 2).

La evaluación de los resultados de la FVCPD mantiene una coherencia con la metodología activa empleada. Además, dado que el EEES insta a realizar una evaluación por competencias, hemos considerado las indicaciones de expertos (Baartman, Bastiaens, Kirschner y Van der Vleuten, 2006; Valverde y Ciudad, 2014) para diseñar una evaluación sostenible y orientada al aprendizaje, integrando para ello estrategias (medios, herramientas y técnicas de evaluación) que suponen oportunidades de desarrollo competencial en el presente académico y el futuro laboral (Briones, GómezLinares y Palomera, 2015). Por tanto, también es una evaluación formativa y sumativa, al ofrecer ejemplos de resolución (e.g. de las tareas 2 y 3), instrumentos de evaluación (i.e. rúbricas con el 
grado de presencia de cada criterio de evaluación); feedback de resultados y orientaciones de mejora (en especial de la Tarea 1, al ser sistemática y continua); y contemplar técnicas de evaluación (i.e. auto-informes de la propia actuación y de la experiencia). Además, se prescinde de examen final de contenidos teóricos, siendo los medios de evaluación el cuaderno de bitácora individual (20\%), la resolución del dilema moral (25\%), el análisis y resolución de un caso (25\%) y la identificación, análisis y representación de un problema docente (40\%).

Figura 2. Descripción de la secuencia de fases seguidas implementando el Teatro Foro.

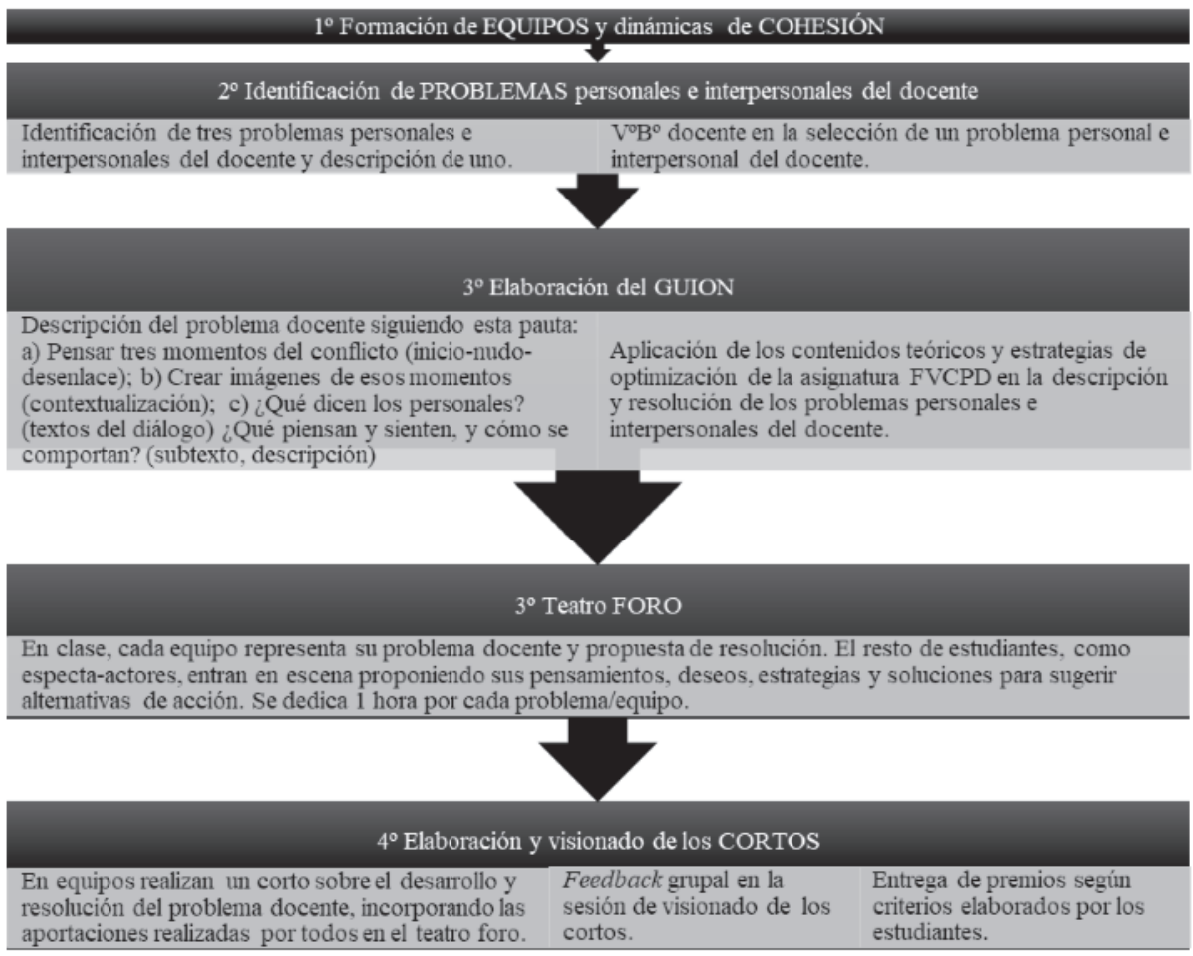

Se presenta el diseño, implementación y evaluación de un programa para una asignatura específica de ASE, integrada en el currículo básico y obligatorio de la formación inicial de docentes con objeto de valorar su eficacia atendiendo a las percepciones de los estudiantes sobre las metodologías integradas en la asignatura y su propio proceso de aprendizaje. Así mismo, se explora el impacto del programa en una de las CES que más atención recibe: la empatía. La hipótesis derivada de la literatura científica es que el alumnado mejora su empatía cognitiva y afectiva tras el proceso de enseñanza-aprendizaje de esta asignatura.

\section{METODOLOGÍA}

\section{Muestra}

En este estudio participaron 314 estudiantes, de los cuales el 35\% son estudiantes del Grado de Magisterio en Educación Infantil (EI) y el 65\% del Grado en Educación Primaria (EP). La media de 


\section{PSICOLOGÍA POSITIVA EN LAS AULAS DE MAGISTERIO CON BITÁCORA, DEBATE, ABP Y TEATRO}

edad de la muestra es 18.93 años ( $D T=3.07$ ), siendo el valor mínimo de 17 años y el máximo de 45 años. El $21 \%$ son hombres y el $79 \%$ restante son mujeres. La prueba $t$ para muestras independientes revela que no hay diferencias significativas en la edad en función del Grado $(t(275)=0.18 ; p=$ .86). Sin embargo, nos encontramos diferencias significativas analizando el Chi cuadrado entre el Grado y el género $\left(\mathrm{Chi}^{2}(1,314)=16.81, p<.01\right)$; siendo el grupo de estudiantes del Grado de El fundamentalmente femenino, como es propio de esta titulación. Del total de la muestra participante, 165 estudiantes participaron en los dos momentos de evaluación (37\% de El, 79.4\% mujeres, 18.82 años de media).

\section{Diseño}

El diseño es longitudinal y transversal, descriptivo y correlacional con objeto de valorar el programa en FVCPD.

\section{Variables e instrumentos}

Meta-conocimiento sobre la experiencia educativa (preferencias y sugerencias de mejora): Se incluyen cuatro preguntas abiertas para que los estudiantes reflexionen e informen sobre el metaconocimiento alcanzado (i.e. lo que han aprendido), sus preferencias (i.e. que es lo que más y menos les ha gustado) y también sobre sus sugerencias para mejorar la metodología y el procedimiento en próximas ediciones.

Meta-conocimiento sobre la contribución de la experiencia educativa en su aprendizaje. Mediante un auto-informe se invita a valorar el grado de utilidad para el propio aprendizaje de cada una de las actividades realizadas empleando una escala Likert de 5 puntos (1 "nada útil"; 5 "muy útil"). Sobre el cuaderno de bitácora se plantea tres cuestiones relativas a cada una de las tareas que comprende (alfa=.79); y en relación al Teatro Foro otras cuatro cuestiones relativas a cada fase (alfa=.77; véanse en la Figura 2). Este instrumento además de ofrecer información, suscita la reflexión de los estudiantes sobre su formación, aprendizaje y desarrollo de las CES entrenadas.

Auto-informe de empatía cognitiva y afectiva (López-Pérez, Fernández-Pinto, y Abad, 2008). Este instrumento constituido por 33 ítems con una modalidad de respuesta Likert de 5 puntos (de 1, "Totalmente en desacuerdo"; 4, "Totalmente de acuerdo") ofrece una medida global de la empatía (alfa $1=.85$; alfa $\left._{2}=.86\right)$ y de cada uno de sus componentes cognitivos y afectivos: Adopción de perspectivas (AP, 8 ítems, alfa $1=.76$; alfa $=.77$ ), Comprensión emocional (CE, 9 ítems, alfa $1=.67$; alfa $_{2}=67$ ), Estrés empático ( $\mathrm{EE}, 8$ ítems, alfa $_{1}=.80$; alfa $_{2}=.79$ ) y Alegría empática ( $\mathrm{AE}, 8$ ítems, alfa $_{1}=.77 ;$ alfa $\left._{2}=.76\right)$.

\section{PROCEDIMIENTO}

Se solicitó la participación voluntaria a los estudiantes, al inicio y al final del primer semestre, explicando el objetivo de la investigación, y su derecho a abandonar la colaboración en cualquier momento. Los estudiantes contestaron de manera individual y anónima (mediante un sistema de códigos personales) al cuestionario en formato online desde sus teléfonos inteligentes en clase, en el horario de la asignatura FVCPD.

El estudio ha salvaguardado las cuestiones éticas, siguiendo el Código de Buenas Prácticas del Consejo Superior de Investigaciones Científicas (CSIC, 2011) y del Comité de Ética de la Investigación en Humanidades y Ciencias Sociales de la UC.

\section{ANÁLISIS DE DATOS}

Los datos cuantitativos se han analizado empleando el programa SPSS 22.0.; mientras que para identificar las categorías emergentes en los datos cualitativos se ha empleado el análisis de contenido con la triangulación de investigadores (Ruiz, 2003). 


\section{RESULTADOS}

En primer lugar, se aportan los resultados vinculados con la percepción del alumnado, es decir, su meta-conocimiento sobre la experiencia educativa (preferencias y sugerencias); así como sobre su impacto en el aprendizaje. Por último, se presentan los resultados del impacto de la FVCPD sobre la empatía, y la relación entre las variables cuantitativas del estudio.

Meta-conocimiento sobre la asignatura: preferencias, sugerencias e impacto percibido sobre el aprendizaje

En las siguientes Figuras se presentan las categorías emergentes del análisis de contenido realizado sobre las respuestas de los estudiantes a las cuestiones sobre sus preferencias (¿Qué te ha gustado más?, ¿qué te ha gustado menos?, Figuras 3 y 4, respectivamente), sus aprendizajes (¿Qué has aprendido?, Figura 5) y sus sugerencias para optimizar metodológicamente la asignatura (Figura 6).

Figura 3. Categorías emergentes para la pregunta sobre lo más valorado de la FVCPD.

\begin{tabular}{|c|c|c|}
\hline $\begin{array}{l}\text { Categorías } \\
\text { (etiquetas y descripción) }\end{array}$ & Ejemplos & $f$ \\
\hline Teoría-contenidos (general) & "los temas que tratado, me han parecido bastante interesantes" & 3 \\
\hline Valores & "el tema de valores porque me hizo conocerme más a mi la misma" & 5 \\
\hline Empatía & "Conocer técnicas para empatizar mejor" & 16 \\
\hline Resolución conflictos & $\begin{array}{l}\text { "Aprender métodos y actitudes para entender a los demás y solucionar } \\
\text { conflictos" }\end{array}$ & 8 \\
\hline Emociones/Regulación & $\begin{array}{l}\text { "He aprendido muchísimo sobre técnicas de regulación de emociones y, } \\
\text { debido a mi situación personal, me han servido de mucho" }\end{array}$ & 30 \\
\hline Autoestima Sana & $\begin{array}{l}\text { "El tema de la autoestima, para poder ponerlo en práctica con gente } \\
\text { cercana que necesita ayuda" }\end{array}$ & 17 \\
\hline Escucha activa & "Me ha gustado mucho hablar sobre la escucha activa" & 6 \\
\hline Asertividad & "Me ha gustado más la asertividad", & 3 \\
\hline Dinámicas de clase/prácticas & $\begin{array}{l}\text { "Que se trata de una asignatura en la que la teoria se desarrolla mediante } \\
\text { ejemplos" }\end{array}$ & 28 \\
\hline Tarea 1. Bitácora & $\begin{array}{l}\text { "El auto-estudio y la auto-comprensión que realizamos al hacer las tareas } \\
\text { del cuaderno de bitácora, ya que tienes la opción de estudiarte a ti mismo } \\
\text { y ver en lo que puedes mejorar" }\end{array}$ & 6 \\
\hline Tarea 2. Dilemas Morales & $\begin{array}{l}\text { "La elaboración del DM me ha ayudado bastante a clarificar mi jerarquia } \\
\text { de valores y lo considero lo más útil de la asignatura" }\end{array}$ & 11 \\
\hline Tarea 3. ABP & $\begin{array}{l}\text { "Realizar la tarea } 3 \text { primero en clase cada uno aportando sus ideas } \\
\text { principales y luego completarlo individualmente" }\end{array}$ & 5 \\
\hline Tarea 4. TF (en general) & $\begin{array}{l}\text { "Me ha gustado más la elaboración del TF, pues con esa actividad práctica } \\
\text { hemos puesto en ejecución lo aprendido en la asignatura" }\end{array}$ & 64 \\
\hline Representación & $\begin{array}{l}\text { "Quizás, la representación teatral por primera vez ya que, aparte de } \\
\text { experimentar sensaciones nuevas he podido ponerme en la piel de un } \\
\text { personaje diferente a mi"” }\end{array}$ & 7 \\
\hline Grabación video & "Lo que más me ha gustado ha sido la grabación del video"” & 16 \\
\hline Método de evaluación & $\begin{array}{l}\text { "Los trabajos, es una manera de representar el trabajo diario sin } \\
\text { exámenes" }\end{array}$ & 2 \\
\hline Autoconocimiento & $\begin{array}{l}\text { "Ser capaz de darme cuenta de cosas que desconocía de mí y que no tenía } \\
\text { tan en cuenta" }\end{array}$ & 4 \\
\hline \multicolumn{3}{|l|}{ Metodología vivencial: } \\
\hline $\begin{array}{r}\text { Clima positivo, cohesivo, } \\
\text { dinámico }\end{array}$ & $\begin{array}{l}\text { "Actividades para conocernos unos a otros y que haya un ambiente más } \\
\text { grupal a la hora de trabajar" }\end{array}$ & 12 \\
\hline Trabajar en grupo & $\begin{array}{l}\text { "Me ha gustado la interacción con mis compañeros ya que en otras } \\
\text { asignaturas no hemos tenido tanta oportunidad" }\end{array}$ & 6 \\
\hline Estilo docente & $\begin{array}{l}\text { "Lo que más me ha gustado ha sido la forma y la dinámica de la profesora. } \\
\text { Sobre todo, su forma de trasmitir los conocimientos" }\end{array}$ & 4 \\
\hline Otros & "Todas las actividades me han servido, muy útiles y divertidas" & 4 \\
\hline
\end{tabular}


Figura 4. Categorías emergentes para la pregunta sobre lo menos valorado de la FVCPD.

\begin{tabular}{|c|c|c|}
\hline $\begin{array}{l}\text { Categorías } \\
\text { (etiquetas y descripción) }\end{array}$ & Ejemplos & $f$ \\
\hline \multirow{2}{*}{$\begin{array}{l}\text { Teoría-Contenidos } \\
\text { (general) } \\
\\
\text { Trabajo en equipo }\end{array}$} & $\begin{array}{l}\text { "Tal vez el grueso teórico si que se me hizo un poco pesado pero al fin y al } \\
\text { cabo no deja de ser interesante" }\end{array}$ & 12 \\
\hline & $\begin{array}{l}\text { "Teoría sobre el trabajo en equipo, creo que es importante pero me no } \\
\text { parecia tan atrayente" }\end{array}$ & 2 \\
\hline Autoestima sana & $\begin{array}{l}\text { "El tema de la Autoestima Sana. No porque no sea de interés, sino porque } \\
\text { cuando hice la tarea } 1, \text { resultó la más complicada de todas" }\end{array}$ & 4 \\
\hline Comunicación & "El tema de la comunicación porque era igual que en Lengua" & 1 \\
\hline Valores y toma de decisiones & "Valores y toma de decisiones, pero también me ha gustado" & 5 \\
\hline Regulación emocional & "La regulación emocional”; "Los ejercicios de relajación”, & 2 \\
\hline Formación del docente & "Del tema 1, algún aspecto más teórico de la formación de docentes" & 5 \\
\hline Asertividad y Res. conflictos & "La parte de asertividad", "El último tema", & 2 \\
\hline Tarea 1. Bitácora & $\begin{array}{l}\text { "Quizás el cuaderno de bitácora por su gran extensión pero reconozco que es } \\
\text { de vital importancia ya que además no hay un examen final"” }\end{array}$ & 21 \\
\hline Tarea 2. Dilemas morales & "Los dilemas, habia mucho que abarcar e identificar" & 16 \\
\hline Tarea 3. ABP & "El análisis de caso puesto que me costó bastante realizarlo" & 18 \\
\hline Tarea 4. TF (en general) & $\begin{array}{l}\text { "El TF fue una experiencia positiva aunque me gustaria haber sacado más } \\
\text { aprendizajes de ella" }\end{array}$ & 9 \\
\hline Elaboración del guion & "Guion y edición del teatro" & 1 \\
\hline Representación & "La dificultad de representar el TF en clase, debido a la vergüenza" & 9 \\
\hline Grabación y edición & "Grabar el video ya que hemos tenido muchas dificultades" & 6 \\
\hline Lecturas & "Las lecturas obligatorias que habia que leerse para hacer la tarea 1", & 7 \\
\hline $\begin{array}{l}\text { Metodología vivencial } \\
\qquad \text { Clima, participación }\end{array}$ & $\begin{array}{l}\text { "Cuando compañeros no han querido enriquecer con sus aportaciones al } \\
\text { resto." "Cuando las clases eran muy monótonas y me aburria" }\end{array}$ & 11 \\
\hline Trabajo en grupo & "Demasiados trabajos en grupo. Es dificil coincidir en tiempo libre" & 7 \\
\hline Estilo docente & $\begin{array}{l}\text { "La manera de impartir la asignatura y a su misma vez de explicarla" } \\
\text { "Algunos power point" }\end{array}$ & 4 \\
\hline Dinámicas en clase & $\begin{array}{l}\text { "Algunas dinámicas para hacer en público pero por la vergüenza no por la } \\
\text { dinámica en sí" }\end{array}$ & 7 \\
\hline Evaluación: & "La entrega de todos los trabajos al final y no al final de cada tema" & 4 \\
\hline Sobrecarga de tareas & "Tener que realizar tantas tareas" & 14 \\
\hline Dudas al realizar las tareas & $\begin{array}{l}\text { "Entender en un primer momento en que consistian las tareas a realizar" } \\
\text { "La falta de referencia para hacer el cuaderno de bitácora" }\end{array}$ & 7 \\
\hline Subjetividad & $\begin{array}{l}\text { "El método de evaluación podría ser más flexible, es decir, es muy dificil } \\
\text { valorar una opinión y ponerla una nota" }\end{array}$ & 2 \\
\hline Planificación & "No tener tanto tiempo para profundizar en algunos temas" & 10 \\
\hline Satisfacción & "Todo lo visto en la asignatura me parece necesario para el docente" & 15 \\
\hline Otros & $\begin{array}{l}\text { "Algunas ideas repetidas", "Tener que dejar de lado mi timidez tan de } \\
\text { repente, ya que me fue dificil dejar de tener miedo al ridiculo" }\end{array}$ & 6 \\
\hline
\end{tabular}


Figura 5. Categorías emergentes para la pregunta sobre lo aprendido en la asignatura de FVCPD.

\begin{tabular}{|c|c|c|}
\hline $\begin{array}{c}\text { Categorías } \\
\text { (etiquetas y descripción) }\end{array}$ & Ejemplos & $f$ \\
\hline \multicolumn{3}{|l|}{ Valores y toma de decisiones } \\
\hline Identificar valores y contravalores & "saber identificar y analizar valores y contravalores" & 32 \\
\hline Ser tolerante & "...sobre todo a ser más tolerante" & 2 \\
\hline Analizar situaciones y tomar decisiones & "Cómo poder valorar y tomar decisiones de forma más fácil" & 4 \\
\hline \multicolumn{3}{|l|}{ Conciencia de uno mismo y Autoestima } \\
\hline Conciencia de uno mismo & $\begin{array}{l}\text { "He aprendido que debería parame a pensar más sobre mí } \\
\text { misma y cómo quiero ser como persona, mis valores, actitudes y } \\
\text { emociones" }\end{array}$ & 35 \\
\hline Autoestima & $\begin{array}{l}\text { "Esta asignatura me ha ayudado a ser mejor persona, a } \\
\text { valorarme más y a intentar ver mi mejor versión." }\end{array}$ & 28 \\
\hline Autogestión: Autorregulación emocional & $\begin{array}{l}\text { "...ciertas cosas que no sabía, como son los comportamientos } \\
\text { para mejorar tu ira, para tranquilizarse..." }\end{array}$ & 44 \\
\hline \multicolumn{3}{|l|}{ Conciencia social } \\
\hline Empatía & $\begin{array}{l}\text { "Ahora me doy más cuenta de cómo se sienten las personas que } \\
\text { me rodean" }\end{array}$ & 53 \\
\hline Comunicación & $\begin{array}{l}\text { "mejorar mis capacidades de comunicación y comprensión con } \\
\text { las personas que me rodean" }\end{array}$ & 2 \\
\hline Escucha Activa & "He aprendido cómo escuchar a las personas" & 10 \\
\hline \multicolumn{3}{|l|}{ Habilidades de relación } \\
\hline Asertividad & $\begin{array}{l}\text { "...la grabación del teatro foro me ha ayudado a perder cierta } \\
\text { timidez." }\end{array}$ & 16 \\
\hline Trabajo en equipo & "instrumentos para trabajar en equipo" & 3 \\
\hline Resolución de conflictos & $\begin{array}{l}\text { "He aprendido diversas maneras de lidiar con distintos } \\
\text { problemas o situaciones que se sucederán a lo largo de nuestra } \\
\text { vida y nunca nos habían enseñado a como sobrellevarlas" }\end{array}$ & 33 \\
\hline Mejorar mi relación con las personas & "... a mejorar mi relación con las personas cercanas a mi" & 6 \\
\hline \multicolumn{3}{|l|}{ Otros aprendizajes } \\
\hline Herramientas útiles para la vida personal & $\begin{array}{l}\text { "Las formas de poder tratar ciertos cosas que se dan en mi } \\
\text { dia" }\end{array}$ & 23 \\
\hline Competencias personales como docentes & $\begin{array}{l}\text { "He aprendido a identificar las competencias } \\
\text { socioemocionales que deben tener los docentes para poder ser } \\
\text { buenos maestros" }\end{array}$ & 19 \\
\hline
\end{tabular}


Figura 6. Categorías emergentes para la pregunta sobre las sugerencias para optimizar la metodología de la FVCPD.

\begin{tabular}{|c|c|c|}
\hline $\begin{array}{c}\text { Categorías } \\
\text { (etiquetas y descripción) }\end{array}$ & Ejemplos & $f$ \\
\hline $\begin{array}{l}\text { Modificaciones en la Metodología } \\
\text { Docente: } \\
\text { Propuestas de más actividades, } \\
\text { modificaciones en algunas actividades, } \\
\text { y atención a determinados contenidos } \\
\text { teóricos. }\end{array}$ & $\begin{array}{l}\text { "Creo que se debería fomentar no solo la autoestima, sino } \\
\text { también la igualdad de género y la inserción de las personas } \\
\text { de otros países en el nuestro" } \\
\text { "Me gustaría que en parte fuera una asignatura más teórica } \\
\text { porque me he dado cuenta que es una asignatura más } \\
\text { importante de lo que pensaba" }\end{array}$ & 24 \\
\hline $\begin{array}{l}\text { Modificaciones en la Evaluación: } \\
\text { Sobre los instrumentos de evaluación y } \\
\text { explicación de las tareas evaluadas. }\end{array}$ & $\begin{array}{l}\text { "Las rúbricas pueden presentarse de otra forma, es decir, de } \\
\text { una forma más clara y mostrando principalmente lo que se pide } \\
\text { en concreto a la hora de elaborar un trabajo" } \\
\text { "Una mejor explicación del cuaderno de bitácora dónde } \\
\text { exista una modelo/ejemplo a seguir con lo que se pide y con lo } \\
\text { que no" }\end{array}$ & 13 \\
\hline $\begin{array}{l}\text { Satisfacción con la FVCPD: } \\
\text { No se contempla la necesidad de } \\
\text { modificar nada. }\end{array}$ & $\begin{array}{l}\text { "Me gustaria que los siguientes cursos siguieran haciendo } \\
\text { estos debates y el video de la obra porque son actividades que } \\
\text { ninguna asignatura salvo esta propone y me parecen ideas } \\
\text { estupendas" }\end{array}$ & 24 \\
\hline
\end{tabular}

En cuanto a la percepción de los estudiantes sobre la contribución de la experiencia educativa en su aprendizaje, las diferencias de medias $t$ para una muestra con respeto al punto medio de la escala (3) presenta resultados significativos en todos los análisis $(p<.001)$, de tal manera que todas las tareas fueron valoradas de manera positiva.

Figura 7. Representación de las puntuaciones medias en la valoración de la utilidad para el aprendizaje de cada tarea del programa de FVCPD.

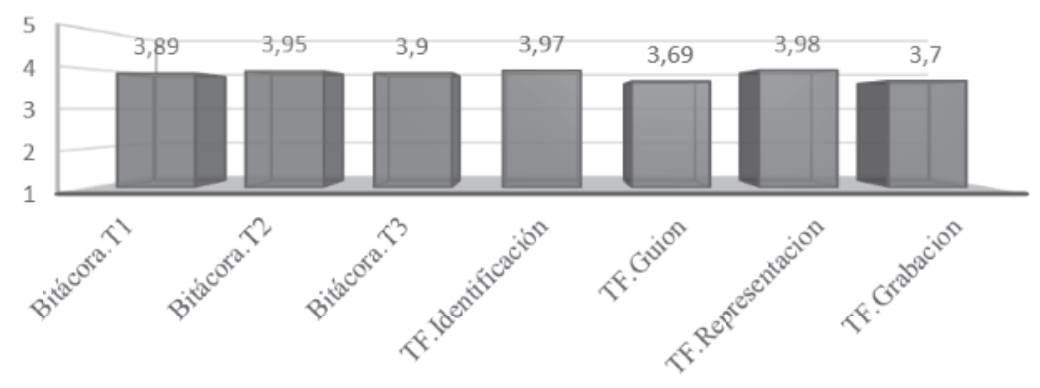

Las diferencias de medias $t$ para muestras relacionadas entre cada par de ítems, revela que hay tareas que se consideraron de mayor utilidad que otras, aunque en términos generales no se observan diferencias al comparar los factores: cuaderno de bitácora y $\operatorname{TF}(t(1,201)=1.25$, n.s.). Las diferencias significativas entre las tareas conciernen a las comparaciones de la elaboración del Guion y de la Grabación del TF con cada una de las tres tareas de la Bitácora $(p<.05)$, y la Identificación del 
caso y su Representación ( $p<.001$ ). Como podemos observar en la Figura 7, las tareas menos valoradas fueron precisamente la elaboración del Guion y la Grabación del corto.

Impacto de la FVCPD sobre la empatía y sus factores

Los análisis realizados (pruebas $t$ para muestras relacionadas) sobre el impacto del programa de FVCPD sobre la empatía, muestran diferencias significativas en uno de sus factores: CE (véase Tabla 1); después del semestre el alumnado fortalece su empatía, reflejada en uno de sus factores cognitivos.

Tabla 1

Descriptivos en la variable empatía y sus factores, y resultados de las pruebas $t$

\begin{tabular}{|c|c|c|c|c|}
\hline \multicolumn{2}{|c|}{ Comparaciones pre-post } & \multirow{2}{*}{$\begin{array}{c}\text { Media } \\
3.84\end{array}$} & \multirow{2}{*}{$\begin{array}{c}\text { Desviación } \\
\text { estándar }\end{array}$} & \multirow{2}{*}{$\begin{array}{c}\text { Pruebas t: } \\
t(1,164)\end{array}$} \\
\hline Par 1 & AP1 & & & \\
\hline & AP2 & 3.85 & 0.50 & $-0.50, n . s$ \\
\hline \multirow[t]{2}{*}{ Par 2} & CE1 & 3.63 & 0.47 & \multirow{2}{*}{$-2.25, p<.05$} \\
\hline & CE2 & 3.71 & 0.46 & \\
\hline \multirow[t]{2}{*}{ Par 3} & EE1 & 3.33 & 0.69 & \multirow{2}{*}{$0.24, n . s}$. \\
\hline & EE2 & 3.32 & 0.70 & \\
\hline \multirow[t]{2}{*}{ Par 4} & $\mathrm{AE} 1$ & 4.32 & 0.42 & \multirow{2}{*}{$1.02, n . s}$. \\
\hline & AE2 & 4.28 & 0.44 & \\
\hline \multirow[t]{2}{*}{ Par 5} & EMPATIA1 & 3.77 & 0.36 & \multirow{2}{*}{$-0.91, n . s}$. \\
\hline & EMPATIA2 & 3.79 & 0.37 & \\
\hline
\end{tabular}

Nota: $n . s .=$ no significativo.

Correlaciones entre las variables del estudio

A la luz de las correlaciones presentadas en la Tabla 2, se puede señalar que las puntuaciones sobre la utilidad de las estrategias de enseñanza-aprendizaje empleadas se relaciona de manera positiva con la empatía en el segundo momento, y con sus factores. Destaca que la utilidad percibida de las tareas 2 y 3 del cuaderno de bitácora se relacionen con todos los factores de la empatía, mientras que las tareas de identificación y guion lo hagan con todos los componentes menos con la CE, la representación solo con los componentes emocionales, y la grabación con el estrés empático.

Tabla 2

Correlaciones entre las variables cuantitativas del estudio

\begin{tabular}{lccccccc}
\hline & T1 & T2 & T3 & Id. caso & Guion & Represent. & Grabación \\
\hline AP2 & $.18^{*}$ & $.24^{* *}$ & $.27^{* * *}$ & $.18^{* *}$ & $.18^{*}$ & .08 & .13 \\
CE2 & .09 & $.14^{*}$ & $.24^{* *}$ & .10 & .05 & -.01 & .12 \\
EE2 & .13 & $.19^{* *}$ & $.24^{* * *}$ & $.26^{* * *}$ & $.19^{* *}$ & $.16^{*}$ & $.18^{*}$ \\
AE2 & .11 & $.25^{* * *}$ & $.21^{* * *}$ & $.24^{* *}$ & $.16^{* *}$ & $.16^{*}$ & .09 \\
EMPATIA2 & $.18^{*}$ & $.28^{* * *}$ & $.34^{* * *}$ & $.27^{* * *}$ & $.20^{* *}$ & $.15^{*}$ & $.18^{* *}$ \\
\hline
\end{tabular}

Nota: $* \mathrm{p}<.05 ; * * \mathrm{p}<.01 ; * * * \mathrm{p}<.001$ 


\section{CONCLUSIONES}

El programa de la asignatura FVCPD ha mostrado su impacto en la competencia empática de manera global. Análisis más finos permiten señalar que el impacto ha tenido lugar en el componente cognitivo de la misma denominado $\mathrm{CE}$, referido a la capacidad de reconocer y comprender los estados emocionales, las intenciones y las impresiones de las otras personas. Este resultado nos parece coherente puesto que todas las tareas realizadas demandan la puesta en práctica de dicha competencia. Hubiese sido deseable que mejorase también la capacidad intelectual o imaginativa de ponerse en el lugar de otra persona (AP) lo cual implica que, aunque las tareas están diseñadas para la toma de perspectiva, es probable que requieran de mayor incidencia como la tarea 2, relativa al trabajo de dilemas morales, que en ediciones anteriores implicaba tres ejercicios de este tipo. Para ello, convendría realizar de manera más explícita la toma de perspectiva en las tareas 3 y 4.

Por otra parte, ha sido deseable que no incrementase el EE, pues si la capacidad de compartir las emociones negativas de otra persona supone la no desconexión con las mismas, en exceso, pueden sobrevenir situaciones de estrés personal o burnout profesional. Y finalmente, la AE partía de puntuaciones medias muy altas, no presentando así, la necesidad ni oportunidad de mejoría.

Los análisis cualitativos identifican que los principales aprendizajes realizados por el alumnado corresponden mayoritariamente con el desarrollo de la competencia de conciencia social, poniendo en práctica y valorando más la necesidad de la empatía tanto para la vida personal como para la profesional. Por otra parte, destaca la competencia de autogestión emocional y la capacidad de autorregulación a través de técnicas específicas. También es importante el aprendizaje sobre resolución de conflictos, el conocimiento de sí mismo y la identificación de valores y contravalores para la toma de decisiones responsable. Además, los datos señalan un aprendizaje de herramientas útiles para la vida diaria y para su futuro como docentes. Por último, destacar que ocho estudiantes han señalado haber aprendido a "ser mejor personas", que supone un indicador más del aprendizaje en sensibilidad moral alcanzado en este programa.

El resto de resultados alcanzados permiten la identificación de elementos clave para la optimización del programa. Por una parte, la valoración realizada de los componentes esenciales de cada metodología sugiere cuál requiere de un mayor seguimiento compartido entre profesorado y alumnado. Como ofrecer ejemplos de resolución de todas las tareas, dado que en el programa considerado se ofrecían tan sólo de las tareas 2 y 3 . En próximas ediciones se pueden mostrar buenos ejemplos y errores más comunes que sirvan para ilustrar todas las tareas requeridas para la evaluación de la asignatura. Además, para aliviar la sobrecarga percibida y las dudas en la realización de las tareas se plantea la fusión de las tareas 3 y 4 (eliminando la grabación de los cortometrajes) y la optimización de las rúbricas, haciéndolas más comprensibles para el alumnado.

Por otra parte, aunque el alumnado reclame formación eminentemente práctica, también es cierto que las propuestas específicas realizadas para la formación de maestros en esta materia, contempla junto con una formación personal vivenciada, una formación teórica en el marco conceptual de las emociones, competencias socio-emocionales, su desarrollo y educación (e.g. Bisquerra, 2005; Fernández Domínguez et al., 2009).

Finalmente, los resultados alcanzados son satisfactorios, pero también nos instan a seguir investigando con objeto de optimizar las herramientas metodológicas empleadas, para la docencia y para la evaluación, dada la importancia de desarrollar competencias emocionales en los estudiantes universitarios (Del Rosal, Dávila, Sánchez, y Bermejo, 2016).

\section{REFERENCIAS BIBLIOGRÁFICAS}

ANECA (2004). Libro Blanco. Título de Grado en Magisterio, Vol. 1. Recuperado de: http://g0o.gl/s22zbj 
Baartman, L. K. J., Bastiaens, T. J., Kirschner, P. A., y Van Der Vleuten, C. P. M. (2006). The wheel of competency assessment: Presenting quality criteria for competency assessment programs. Studies in Educational Evaluation, 32(2), 153-170. doi:10.1016/j.stueduc.2006.04.006

Bisquerra, R. (2005). La educación emocional en la formación del profesorado. Revista Interuniversitaria de Formación del Profesorado, 19(3), 95-114.

Briones, E. y Gómez-Linares, A. (2016). Psicología para Docentes: Guía y casos resueltos aplicando el Aprendizaje Basado en Problemas (ABP). Madrid: Pirámide.

Briones, E. y Lara, L. (2016). Educación ética en la Universidad a través del diálogo multicultural online. Comunicar, 47(XXIV), 99-107. doi:10.3916/c47-2016-10

Briones, E., Gómez-Linares, A., y Palomera, R. (2015). La formación en valores en el EEES: propuesta de instrumento, medio y técnicas de evaluación. En N. González, I. Salcines, y E. García (Coords), Tendencias emergentes en evaluación formativa y compartida en docencia. El papel de las nuevas tecnologías (pp. 215-233). Santander: Editorial Universidad de Cantabria.

CASEL, Collaborative for Academic, Social, and Emotional Learning (2005). Safe and sound: An educational leader's guide to evidence-based social and emotional learning programs-Illinois edition. Chicago, IL: Collaborative for Academic, Social, and Emotional Learning.

Durlak, J. A., Weissberg, R. P., Dymnicki, A. B., Taylor, R. D., y Schellinger, K. B. (2011). The impact of enhancing students' social and emotional learning: A meta-analysis of school-based universal interventions. Child Development, 82(1), 405-432.

Del Rosal, I., Dávila, M.A., Sánchez, S., y Bermejo, M.L. (2016). La inteligencia emocional en estudiantes universitarios: diferencias entre el Grado de maestro en educación primaria y los grados en ciencias. International Journal of Developmental and Educational Psychology, INFAD Revista de Psicología, 1(2), 51-62. doi: 10.17060/ijodaep.2016.n1.v2.176

Fernández Domínguez, M. R. (2005). Más allá de la educación emocional. La formación para el crecimiento y desarrollo personal del profesorado. PRH como modelo de reverencia. Revista Interuniversitaria de Formación del Profesorado, 19(3), 195-251. Recuperado de https://dialnet.unirioja.es/descarga/articulo/2126781.pdf

Fernández Domínguez, M. R., Palomero, J. E., y Teruel, M. P. (2009). El desarrollo socioafectivo en la formación inicial de los maestros. Revista Electrónica Interuniversitaria de Formación del Profesorado, 12(1), 33-50. Recuperado de http://goo.gl/Aip5Cn

Grupo de Psicología Positiva Aplicada (2017). Manual de ejercicios de Psicología Positiva aplicada. Ejercicios sencillos para incrementar el bienestar. Madrid: COP de Madrid. (https://g00.gl/teVrNK).

Kornacki, S. A. y Caruso, D. R. (2007). A theory-based, practical approach to emotional intelligence training: ten ways to increase emotional skills. En J. Ciarrochi y J. Mayer (Eds.), Applying Emotional Intelligence: A Practitioner's Guide (pp. 53-88). New York: Psychology Press.

López-Pérez, B., Fernández-Pinto, I., y Abad. F. J. (2008). Test de empatía cognitiva y afectiva, TECA. Madrid: TEA.

Moreno, B. y Gálvez, M. (2010). La Psicología Positiva va a la escuela. Típica, Boletín Electrónico de Salud Escolar, 6(1), 210-220. Recuperado de http://g00.gl/8YNqCg

Motos-Teruel, T. y Navarro-Amorós, A. (2012). Estrategias del Teatro del Oprimido para la formación permanente del profesorado. Magis, Revista Internacional de Investigación en Educación, 4(9), 619-635. Recuperado

de https://revistas.javeriana.edu.co/index.php/MAGIS/article/view/3579/2693

Palomera, R., Briones, E., Gómez-Linares, A., y Vera. J. (2017). Filling the gap: improving the social and emotional skills of pre-service teachers. Revista de Psicodidáctica, 22(2), 142 - 149. doi: 


\section{PSICOLOGÍA POSITIVA EN LAS AULAS DE MAGISTERIO CON BITÁCORA, DEBATE, ABP Y TEATRO}

10.1016/j.psicod.2017.05.002

Palomera, R., Briones, E., y Gómez-Linares, A. (2017). Diseño, desarrollo y resultados de un programa de Educación Socio-Emocional para la formación de docentes a nivel de Grado y Postgrado. Contextos Educativos. Revista de Educación, 20, 165-182. doi:10.18172/con.2988

Pañellas, M., Alguacil, M., y Boqué, M.C. (2016). Afectos y cognición de los futuros maestros en la resolución de problemas matemáticos. International Journal of Developmental and Educational Psychology, INFAD Revista de Psicología, 1(1), 431-442. doi:10.17060/ijodaep.2016.n1.v1.163

Ruiz, J. I. (2003). Técnicas de triangulación y control de calidad en la investigación socioeducativa. Bilbao: Universidad de Deusto.

Seligman, M.E.P., Ernst, R.M., Gillham, J., Reivich, K., y Linkins, M. (2009). Positive education: positive psychology and classroom interventions. Oxford Review of Education, 35(3), 293-311. D0I: $10.1080 / 03054980902934563$

Valverde, J. y Ciudad, A. (2014). El uso de e-rúbricas para la evaluación de competencias en estudiantes universitarios. Estudio sobre fiabilidad del instrumento. REDU, Revista de Docencia Universitaria, 12 , 49-79. Recuperado de https://dialnet.unirioja.es/descarga/articulo/4691792.pdf 